\title{
Procedimientos de Ensayo para Conexiones tipo Clavija en Estructuras de Madera
}

\author{
Manuel Domínguez ${ }^{*}$, José G. Fueyo, José L. Henares y José A. Cabezas \\ Departamento de Ingeniería Mecánica, Universidad de Salamanca, Avda. de Requejo 33, Zamora-España \\ (e-mail: mdominguez1@usal.es)
}

Recibido Oct. 29, 2013; Aceptado Dic. 4, 2013; Versión final recibida Feb. 6, 2014

\begin{abstract}
Resumen
El objetivo de este trabajo es definir los procedimientos de ensayo a seguir en uniones del tipo clavija para estructuras de madera, a fin de identificar su evolución resistiva en situaciones reales. Se desarrolla una metodología en consonancia con las nuevas directrices de la normativa europea, sobre probetas de tamaño real, reflejando los parámetros principales a tener en cuenta durante el ensayo y la evolución de gráficas tensión-deformación. Se contemplan factores como el aplastamiento de la madera, la flexión de la clavija y el efecto soga. Partiendo del análisis de prototipos, se fabrican herrajes específicos y varias series de probetas, para desarrollar ensayos sobre tres variantes. A saber, unión con bulón roscado, unión con pernos encolados y unión con arandela-tuerca, con el objetivo de analizar los comportamientos del nudo.
\end{abstract}

Palabras clave: madera, ensayos, conexiones, unión, clavija, estructuras.

\section{Test Procedures for Dowel type Connections in Timber Structures}

\begin{abstract}
The aim of this paper is to define the test procedures to be followed in dowel-type joints of timber structures, in order to identify their resistant behavior in real situations. A new methodology is developed according to the new European regulations guidelines on real-size specimens, reflecting the main parameters to take into account during the tests and the evolution of the stress-strain relationships. Factors such as wood crushing, dowel bending and rope effect have been taken into account. From the analysis of prototypes, specific fittings and several series of tests on specimens have been prepared to develop the tests with three options. These are: joint with threaded bolt, joint with glued-in rod and joint with washers and nuts, all this with the objective of analyzing the behavior of the different types of connection.
\end{abstract}

Keywords: timber, lab tests, connections, joint, dowel, structures 


\section{INTRODUCCION}

Los ensayos sobre uniones de madera tipo clavija suponen una problemática compleja debido a la ortotropía y heterogeneidad de la madera y a la influencia sobre la misma de otras variables del entorno (humedad, tiempo de aplicación de la carga, combinación de distintos materiales, patologías, etc.). En términos generales, la normativa ha intentado hacer un análisis independiente de las variables de fallo: aplastamiento de la madera y flexión de la clavija. Es usual que las normas de algunos países tengan un referente claro en las normas más antiguas con experiencia reconocida en dicho campo. En este contexto se pueden citar como ejemplos las normas ANSI de los EEUU, o las normas COPANT en Sudamérica, como modelo de unificación en esta materia (Ariza, 2002). En el ámbito europeo, las normas tradicionales pertenecían al llamado "enfoque de las tensiones básicas" (probetas de tamaño pequeño y en general libres de defectos), basadas en el trabajo con materiales homogéneos como acero y hormigón. Sin embargo, es necesario un tratamiento más cercano a la realidad heterogénea de la madera estructural y su composición. La nueva normativa de ensayos europea aspira a unificar los procedimientos en todos los países de la Unión Europea, con lo cual las normativas nacionales se hallan en proceso de revisión y, por lo tanto, de investigación y mejora.

Para las bases teóricas y prácticas del trabajo con madera en estructuras, se mantiene como referencia el Eurocódigo 5 y sus adaptaciones en cada país, como la norma UNE EN 1995, en el caso de España. Para generar un lenguaje común en el tratamiento de la madera de uso estructural, se ha definido en la Unión Europea un sistema denominado de clases resistentes, recogido en la norma UNE EN 338 (Hermoso et al., 2007) que debe ser considerado también en los ensayos. EI CTE DB SE-M clasifica la madera en dos grupos: aserrada de tipo conífera (representada con la letra "C") y frondosa o angiosperma (con la letra "D"). Los diferentes tipos de madera se tipifican mediante dicha letra, seguida de la resistencia característica a flexo-tracción expresada en MPa $\left(\mathrm{N} / \mathrm{mm}^{2}\right)$. En el Reino Unido también se han desarrollado ensayos basados en el Eurocódigo 5 para uniones con clavijas en cortadura doble y encoladas (Davis y Claisse, 2000).

Han sido revisadas y actualizadas normas como la UNE 56544, "Clasificación visual de la madera aserrada para uso estructural. Madera de coníferas", dando lugar a una clasificación en calidades estructurales en base a los defectos comunes antes citados, donde las calidades más bajas suponen el "rechazo" para su uso estructural. Trabajos realizados sobre las propiedades resistentes, referidas a la clasificación visual, indican altos porcentajes de rechazo en casos reales que sugieren cambios en la normativa actual (Íñiguez, 2007). A la madera usada en los ensayos de uniones estructurales le deben ser extensibles estas consideraciones. Se desarrollan condiciones de muestreo e interpretación en la norma UNE EN 384, "Madera con uso estructural. Determinación de los valores característicos de las propiedades mecánicas y densidad", en la cual se establece además la posibilidad de comparar estos ensayos con otros sobre probetas "pequeñas libres de defectos", tal como las cita textualmente. Los ensayos de propiedades mecánicas paralelas a las fibras y densidad en la madera se definen en la norma UNE EN 408.

Gran parte de los ensayos definidos se orientan a determinar las propiedades físicas y mecánicas de la madera estructural de modo independiente (Blanchet, 2004). Por ejemplo, en tracción paralela a la fibra, para obtener el módulo de elasticidad $\mathrm{E}_{\mathrm{t}, 0}$, a compresión paralela a la fibra para calcular el módulo de elasticidad $E_{c, 0}$, de cortante, a flexión, etc. Pero en las estructuras de madera del 20 al $25 \%$ del costo de la estructura está vinculado con el diseño y construcción de las uniones entre sus diferentes elementos que requieren una gran dedicación en tiempo (Argüelles et al., 2003). Se ha estimado que, de un diseño estructural completo, el diseño de la unión, puede representar hasta el $70 \%$ del tiempo total de cálculo para una estructura de madera (Rodd y Leijten, 2003).

Los cálculos de estructuras reales suponen grandes escuadrías de madera, siendo necesario optimizar el diámetro de la clavija para una sección constante de madera y limitar el desplazamiento o deslizamiento de la unión. Las clavijas contribuyen significativamente al coste del sistema de ensamblado estructural (Batchelar y Mcintosh, 1988), de manera que debe cuidarse especialmente el uso de una proporción adecuada de estos elementos. Se buscan sistemas de unión de tipo clavija que traten de reducir el diámetro de la misma, ya que mayores tamaños de estos aumentan las posibilidades de rotura frágil en los componentes de la madera (Guan y Rodd, 2001). El presente artículo aborda un procedimiento acorde con situaciones reales que se pueden dar sobre el nudo y la determinación de su resistencia como unión estructural de madera, en un caso habitual, la unión mediante clavija en dirección transversal, frente a otros como los realizados en dirección longitudinal a la fibra (Bainbridge et al., 2002).

Es necesario obtener valores de resistencias características en los elementos que componen el nudo, en extensión a tensiones en zonas significativas, como ocurría antes, y un mayor nivel de información y confianza en estos ensayos. Se convierte en una necesidad, el empleo de la madera de uso estructural, 
con dimensiones comerciales, certificados de calidad, caracterización de límites a defectos comunes y grados de certidumbre al ensayo. Los ensayos deben definir valores característicos de resistencia definidos a partir del 5 percentil de una población estadística con distribución normal. Se trata de aquel valor que separa los datos de la muestra dejando por debajo un $5 \%$ y por encima el restante $95 \%$. Es decir, solo existe una probabilidad del $5 \%$ de encontrar resistencias menores.

La norma UNE EN 26891, "Estructuras de madera. Uniones realizadas con elementos de fijación mecánicos. Determinación de las características de resistencia y deslizamiento" establece los principios generales que deberán observarse para lograr la compatibilidad de los resultados en las investigaciones llevadas a cabo en diferentes laboratorios. Esta norma es una trascripción de la norma ISO 6891 del año 1983, que ha sido empleada por diversos autores (Awaludin et al., 2008; Dorn et al, 2013). Es aplicable a las uniones realizadas con elementos de fijación mecánicos para estructuras con cargas estáticas. Los principios también pueden emplearse para el ensayo de otras uniones, aunque la misma norma reconoce que pueden ser necesarias modificaciones al procedimiento de ensayo.

El marco de ensayos está orientado a mejorar el rendimiento de la unión de madera con elementos tipo clavija, modificando distintos parámetros como la densidad o el rozamiento (y con ello la redistribución tensional) en la zona de contacto. Se pretende mejorar la fiabilidad en las mediciones sobre la unión teniendo en cuenta que pueden existir distintas técnicas de superposición a la clavija: refuerzos con placas dentadas (Echavarría et al., 2009), expansiones de pernos (Larsen y Leijten, 2000) o inyección de adhesivos en la zona de contacto (Rodd y Leijten, 2003).

Se añaden elementos que distribuyen la tensión de aplastamiento de la madera y la deformación plástica de la clavija. Esto supone analizar la evolución de la relación entre la carga y el deslizamiento en nuevos conjuntos y el desarrollo de procedimientos de ensayo que faciliten la obtención de resultados acordes con las técnicas de construcción empleadas, tales como la unión con tuerca-arandela o la introducción de adhesivo entre clavija y madera, frente a la flexión simple definida en el Eurocódigo 5 y fundamentada en autores que se han encuadrado en la teoría del rendimiento (Johansen, 1949) (Hilson, 1995). Esta teoría ha sido empleada en el cálculo de uniones tipo clavija y fue desarrollada en Europa en la década de 1940 y viene a predecir el límite de carga de uniones con diferentes configuraciones mixtas. En América del Norte se la denominó como "European Yield Model" (Rodd y Leijten, 2003).

\section{MODELOS DE ENSAYO Y REQUISITOS GENERALES}

La unión debe tener un diseño que cumpla una serie de premisas: alta resistencia con baja variabilidad en el tiempo, una rigidez fiable, una elevada ductilidad (que permita la tolerancia a efectos dinámicos, como el sismo), debe ser estable ante cargas con variaciones de temperatura y la humedad, los elementos del conjunto de unión deben afectar lo menos posible a la estética de la estructura (especialmente en construcciones históricas), debe ser de coste reducido y construcción simple, y la posición del elemento de fijación debe condicionar lo menos posible las dimensiones de las vigas. La disposición simétrica permite eliminar excentricidades y componer articulaciones y su uso constructivo es bastante generalizado, por ello se plantea, la unión con clavija en cortadura doble como opción de estudio. Este modelo permite lograr diámetros menores con la superposición de efectos de otras variantes como la modificación del contacto por fricción, la introducción de adhesivo y la fijación con arandela- tuerca.

Los requisitos de los ensayos en las uniones tipo clavija resultan amplios y se agrupan en distintos factores en los cuatro modos de fallo nombrados como $F_{v, r k 1}, F_{v, r k 2}, F_{v, r k 3}$ y $F_{v, r k 4}$ según la figura 1, donde:

i) $F_{v, r k 1}$ es la carga de colapso para el caso de aplastamiento en la madera de las piezas laterales de espesor $t_{1}$ de modo uniforme.

ii) $F_{v, r k 2}$ es la carga de colapso para el caso de aplastamiento en la madera de la pieza central de espesor $t_{2}$ de modo uniforme.

iii) $F_{v, r k 3}$ la carga de colapso para el caso de aplastamiento local en las piezas de madera y fallo por flexión o rótula plástica en el centro de la clavija de diámetro d.

iv) $F_{v, r k 4}$ es la carga de colapso para el caso de aplastamiento local en las piezas de madera y fallo por flexión o rótulas plásticas en el centro y extremos de clavija de diámetro d.

Por ello, el desarrollo de ensayos resulta ciertamente más amplio y complejo debido a los distintos modos de fallo y a las nuevas configuraciones de la clavija que se orientan a mejorar el rendimiento de la unión. 
i)

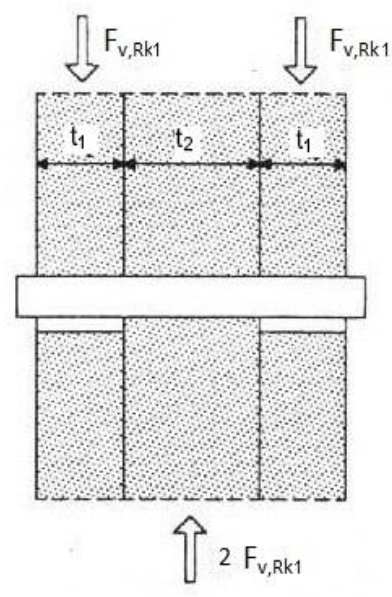

ii)

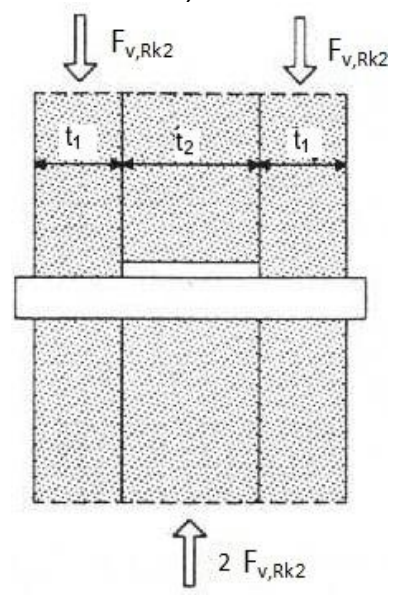

iii)

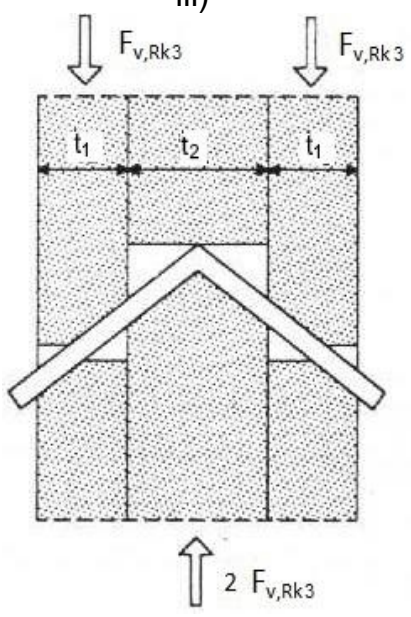

iv)

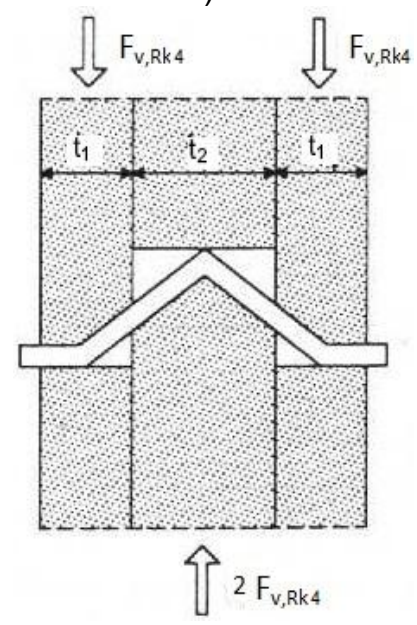

Fig. 1: Modos de fallo en uniones de madera tipo clavija para cortadura doble

\section{PREPARATIVOS PARA EL ENSAYO}

\section{Dispositivos de ensayos}

Se parte de los parámetros descritos en normativas sobre ensayos simples y de caracterizaciones de materiales certificados. El montaje de las probetas sobre la máquina de ensayos debe ser tal que evite la aparición de cualquier otro tipo de esfuerzos secundarios. Bajo esta premisa, se propone como más adecuada una configuración de ensayo de tipo compresión simple, según se representa la figura 2, ya que facilita una distribución uniforme de cargas frente a los problemas que supondría la transmisión de esfuerzos de tracción a la probeta.
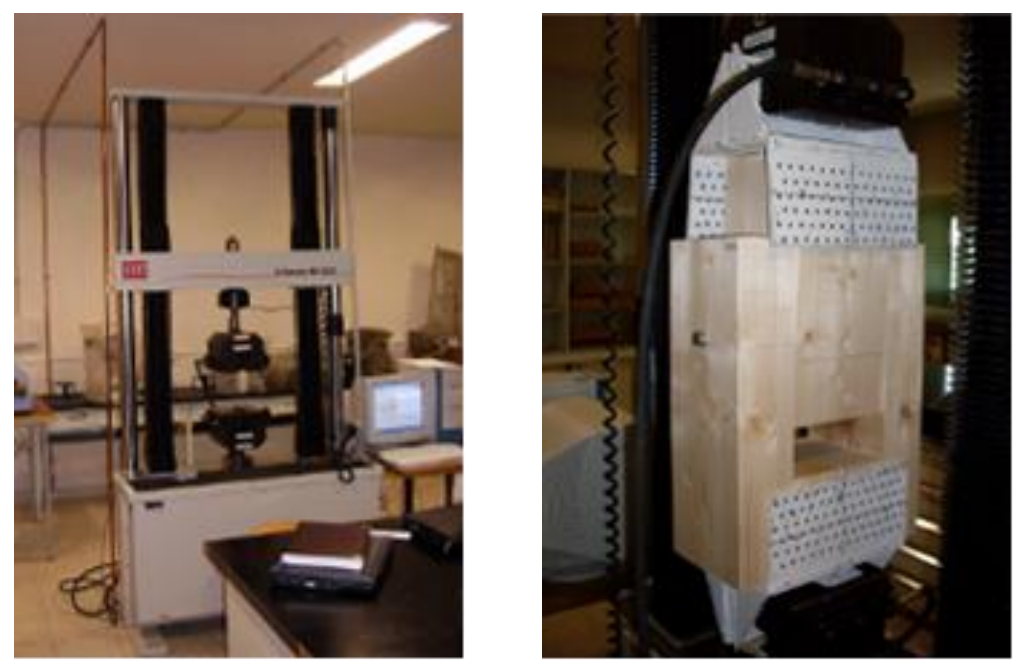

Fig. 2: Máquina y probeta para ensayo de compresión simple

El dispositivo de ensayo según UNE EN 383, "Estructuras de madera: Métodos de ensayo. Determinación de la resistencia al aplastamiento y del módulo de aplastamiento para los elementos de fijación tipo clavija", debe ser tal que no se ejerza entre las placas de acero y las probetas ningún esfuerzo que pueda influir en las medidas, requiriendo:

a) Un dispositivo de carga capaz de aplicar y registrar ésta de forma continua con una precisión de $\pm 1 \%$ de la carga aplicada a la probeta o, para las cargas menores al $10 \%$ de la carga máxima, de $\pm 0,1 \%$ de la carga máxima aplicada a la probeta.

b) Un dispositivo que permita registrar el deslizamiento de los elementos de fijación en la madera con una precisión de $\pm 1 \%$ del deslizamiento, o para los deslizamientos menores de $2 \mathrm{~mm}$, de $\pm 0,02 \mathrm{~mm}$. 
Por razones de costes y fiabilidad, se aconseja la separación de los dispositivos de aplicación de carga y medición de desplazamientos. Esto facilita la flexibilidad para cuantificar medidas, frente a equipos que integran todas las medidas y tienden a rigidizar los ensayos, en ocasiones sin contemplar particularidades constructivas. Los elementos de fijación deben dar lugar a superficies de contacto suficientemente rígidas, placas de acero de alta resistencia de la serie F-114 pueden ser una buena opción. También deben garantizar un contacto homogéneo en la superficie de transmisión de esfuerzos. Antes de la colocación del elemento de fijación, la madera debe acondicionarse en una atmósfera a condiciones constantes, temperatura de $20^{\circ} \mathrm{C} \pm 2^{\circ} \mathrm{C}$ y humedad relativa ambiental del $60 \% \pm 5 \%$.

\section{Control de humedad y parámetros de monitorización}

El acondicionamiento de las probetas debe ser lo más parecido posible al contenido en humedad de equilibrio en condiciones de servicio (Argüelles et al., 2003). En cualquier caso, se recomienda que esté por debajo del 25\% (Wood Handbook, 1998). Lo más aconsejable es emplear cámaras climáticas donde se mide la humedad relativa de la madera, aunque existen otros métodos como el de estufa, que supone llevar a la madera a temperaturas de $103^{\circ} \mathrm{C}$ para conseguir reducciones en el contenido de humedad por desecación. Verificadas las dimensiones, la densidad y el contenido en agua, se volverán a comprobar una vez ejecutado el ensayo. El contenido de humedad obtenido por desecación se puede considerar como previo a la adquisición de la madera, si está certificada, y puede penalizar la resistencia de la madera si se sobrepasa el $12 \%$ de humedad según la norma UNE EN 384. Se debe verificar con equipos como el xilohigrómetro de resistencia, debidamente calibrado. Estos datos son rápidos y fáciles de obtener según la figura 3.
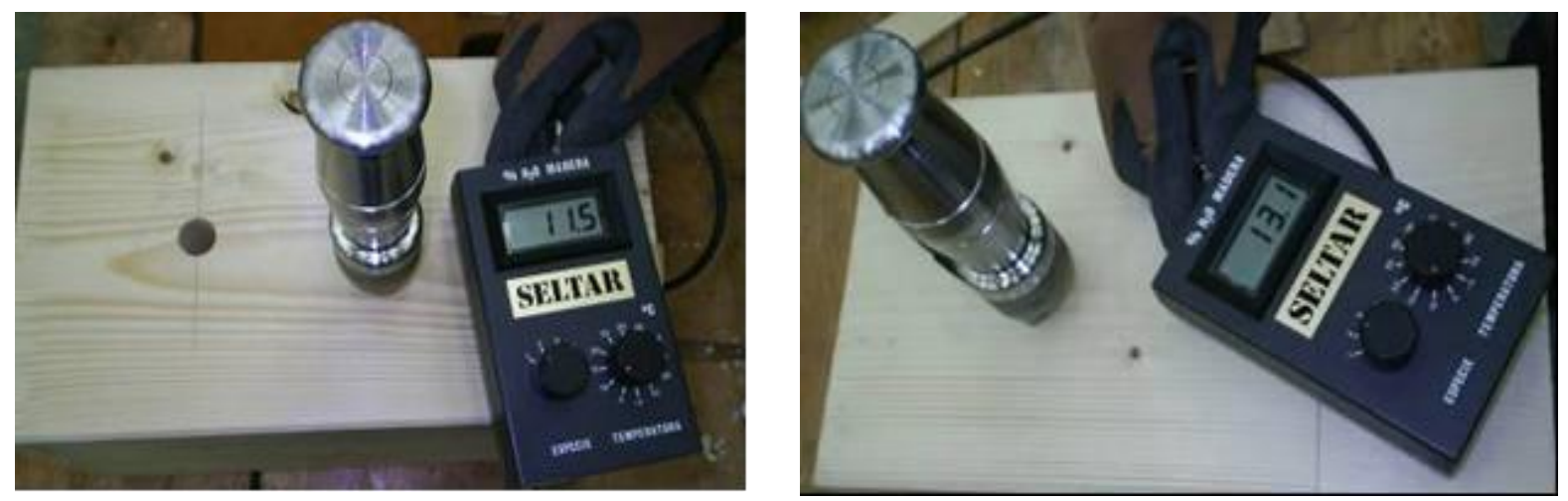

Fig. 3: Caracterización con xilohigrómetro Seltar HGD-1

\section{Caracterización de la madera y clavijas}

Debido a la posterior exigencia para el uso estructural, es muy aconsejable la definición de los tipos de la madera con un marcado de certificación dentro de las reconocidas como clases resistentes. Igualmente para las clavijas se emplean materiales normalizados. De esta forma se obtienen garantías en su caracterización de base, evitando distorsiones posteriores a los resultados del ensayo y asegurando la trazabilidad de los materiales que componen las probetas. Ello, sin perjuicio de la realización complementaria de ensayos de resistencia a tracción en el perno o aplastamiento simple en la madera, o en su defecto de métodos alternativos (preferentemente no destructivos) como los penetrómetros (por ejemplo Pilodym ${ }^{\circledR}$ ), que permitan ratificar las propiedades de la madera y de los elementos de unión.

La geometría de la madera debe ser revisada en el proceso anterior a la composición de la probeta porque las escuadrías comerciales pueden tener secciones ligeramente mayores. Estos incrementos de sección están justificados por mermas en los posteriores procedimientos de cepillado y por tolerancias mayores del proceso de serrado. En estos casos, tales excesos deben ser corregidos por métodos de mayor precisión como el propio cepillado y el fresado por control numérico según la figura 4.

\section{Velocidad de carga durante la ejecución del ensayo}

Dado el carácter cuasi estático que se espera en las cargas reales que actúan sobre las estructuras, se aconseja que la velocidad en el ensayo sea reducida, con una precisión en la medida de la carga del $\pm 1 \%$ y una velocidad de ensayo de $1 \mathrm{~mm} / \mathrm{min}$ que garantice situaciones de carga estáticas (Dong, 2006), de forma que por un lado la aplicación lenta y gradual de las fuerzas haga que los desplazamientos de las partículas del cuerpo ocurran con velocidades muy pequeñas, pudiendo despreciarse la energía cinética producida, y 
por otro lado pueda despreciarse el rozamiento con los enlaces externos. De esta forma el trabajo que realizan las fuerzas externas se invierte en energía de deformación, tal y como presuponen las hipótesis básicas de resistencia de materiales.

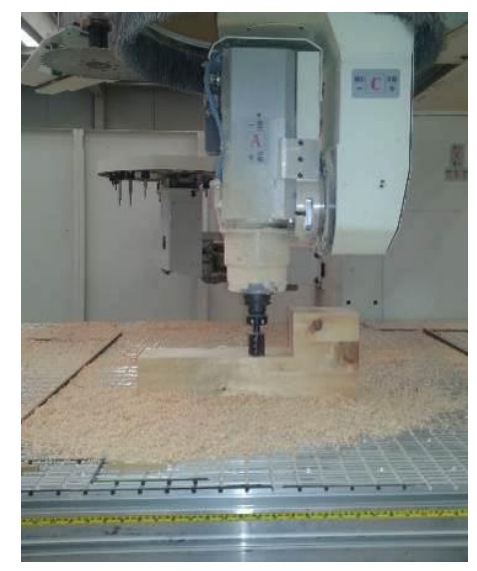

Fig. 4: Fresado por control numérico

Se debe considerar que el elemento de unión puede presentar deslizamiento. Este es limitado en ensayos normalizados como la UNE EN 26891, donde se contemplan velocidades de carga en función del tiempo y descargas de la probeta con tiempos estáticos de asentamiento estudiando el deslizamiento de la unión y limitándolo a $15 \mathrm{~mm}$ para un tiempo total de ensayo de entre 10 a $15 \mathrm{~min}$.

Otro factor a considerar son los posibles sistemas de refuerzo de la clavija, como puedan ser las placas clavo (Echevarría, 2009) o la fuerza de rozamiento en el propio perno, introduciendo modificaciones superficiales que mejoren la adherencia entre éste y la zona de aplastamiento de la madera. Por ejemplo, con elementos roscados (Fueyo et al., 2011) la combinatoria de parámetros puede ser muy amplia: el espesor de piezas de madera, el tipo de material de refuerzo, las direcciones de la fibra de madera de las piezas interior y exterior (Guan y Rodd, 2001).

\section{PROBETAS Y PREPARACION DE ENSAYOS EN UNIONES TIPO CLAVIJA}

\section{Madera}

Se empleó madera aserrada de la clase C24, teniendo especial atención a las certificaciones de calidad. Se trata de una madera de la clase conífera, denominada Picea Abies. Los diversos coeficientes que caracterizan la madera en su comportamiento mecánico son los siguientes: módulos elásticos longitudinales $E_{L}=11000 \mathrm{~N} / \mathrm{mm}^{2}, E_{R}=370 \mathrm{~N} / \mathrm{mm}^{2}, E_{T}=370 \mathrm{~N} / \mathrm{mm}^{2}$, módulos elásticos transversales $\mathrm{G}_{\mathrm{LR}}=690 \mathrm{~N} / \mathrm{mm}^{2}$, $G_{L T}=690 \mathrm{~N} / \mathrm{mm}^{2}, G_{R T}=83,6 \mathrm{~N} / \mathrm{mm}^{2}$, y coeficientes de Poisson $v_{L R}=0,41, v_{L T}=0,41$ y $v_{R T}=0,51$, valores obtenidos por ensayos previos, reconocidos en bibliografía (Argüelles et al., 2003) y según normativa europea UNE EN 338, "Madera estructural. Clases resistentes".

\section{Clavija}

Se optó por varilla roscada de calidad 5.6 zincada. La protección superficial resulta obligada para elementos de fijación según CTE DB SE-M. El Eurocódigo 5 dimensiona la clavija para flexión simple. Aquí la investigación pretende obtener resultados más cercanos a las necesidades prácticas de construcción porque esta superficie roscada facilita el acoplamiento de sistemas tuerca - arandela, de uso muy habitual en estructuras. Además el roscado mejora la adherencia con posibles adhesivos. En algunos casos se han utilizado barras de materiales compuestos (normalmente resina de poliéster reforzada con fibra de vidrio). Estas pueden utilizarse para dar continuidad entre piezas de madera y ocultar la clavija, pero no permiten, lógicamente, la soldadura del acero, aspecto beneficioso de cara a posibles refuerzos en la unión.

Debido a las exigencias de la estética y la continua necesidad de lograr la eficiencia estructural y la competitividad de costos, los métodos de uniones de madera están en constante evolución. Los resultados de ensayos realizados con clavija están orientados a la composición con tuerca-arandela y adhesivos, que potencian la interacción en las intercaras de la madera. Se aconseja que el perno trabaje fundamentalmente bajo situaciones de rótula plástica complementado por efectos de redistribución axil, también llamado efecto 
soga o cable, lo que supone en la práctica que se pueden utilizar clavijas con diámetro entre 8 y $24 \mathrm{~mm}$ para las escuadrías indicadas.

Si se opta por el encolado entre la madera y la barra, puede realizarse según varias posibilidades (Argüelles et al., 2003): 1) Con un taladro en la madera con un diámetro ligeramente superior al de la barra, para conseguir una holgura suficiente para la inyección del adhesivo. Esta diferencia de diámetros es normalmente de $1 \mathrm{~mm}$, aunque otros autores recomiendan de 3 a $5 \mathrm{~mm}$. 2) Con un diámetro del agujero igual al de la barra. El adhesivo se vierte en el agujero y después se introduce a rosca la barra. Para permitir que el adhesivo se distribuya en toda la superficie la barra deberá tener una ranura o canal a lo largo de su longitud normalmente de un tamaño igual a la profundidad del roscado. 3) Otra alternativa es aplicar el adhesivo en la superficie del agujero y en la barra con pincel, no siendo necesario en este caso utilizar barras con ranuras, aunque la resistencia general de la unión será inferior.

El método de montaje es el roscado. Con esta técnica un defecto local del adhesivo no representa una pérdida grave de capacidad, ya que la transmisión de tensiones continúa, de modo mecánico, a través del roscado. Se usarán barras roscadas con la diferencia de diámetros de $1 \mathrm{~mm}$ (Argüelles et al., 2003). Como elemento de fijación adhesiva se empleará resina epoxi (HILTI RE 500), uno de los considerados como apto por el Eurocódigo 5, siempre teniendo en cuenta la clase de servicio y el Documento de Idoneidad Técnica Europeo (DITE) o European Technical Approval (ETA) del adhesivo en cuestión, ya que estos constituyen, por definición, una evaluación técnica favorable de la idoneidad de un producto, ámbito de trabajo y modo de empleo.

\section{Dimensiones de las probetas}

Las piezas de madera son paralelepípedos, figura 5 , que varían en función de las medidas $t_{1}$ y $t_{2}$ (espesor de las piezas de madera). Para los ensayos realizados hasta la fecha, los anchos han sido $t_{1}$ de $70 \mathrm{~mm}$ a $100 \mathrm{~mm}, \mathrm{t}_{2}=200 \mathrm{~mm}$ y diámetros de clavija variable. El valor de $\mathrm{b}$ se mantiene constante en $200 \mathrm{~mm}$. En consideración a la norma UNE EN 383, este valor debe ser mayor o igual a $4 \cdot d$, y las distancias $h_{2}$ y $h_{3}$ deben ser mayores a $5 \cdot d$. Las cotas quedan definidas en la figura 5 y están orientadas a garantizar un comportamiento dúctil de la unión (Echavarria, 2009) y prevenir fallos por cortante o efectos de hienda. Los materiales y geometrías empleados se han tratado en otros estudios, por ejemplo mediante la aplicación del método de los elementos finitos (Fueyo et al., 2011) al objeto de ratificar los resultados con otras técnicas.

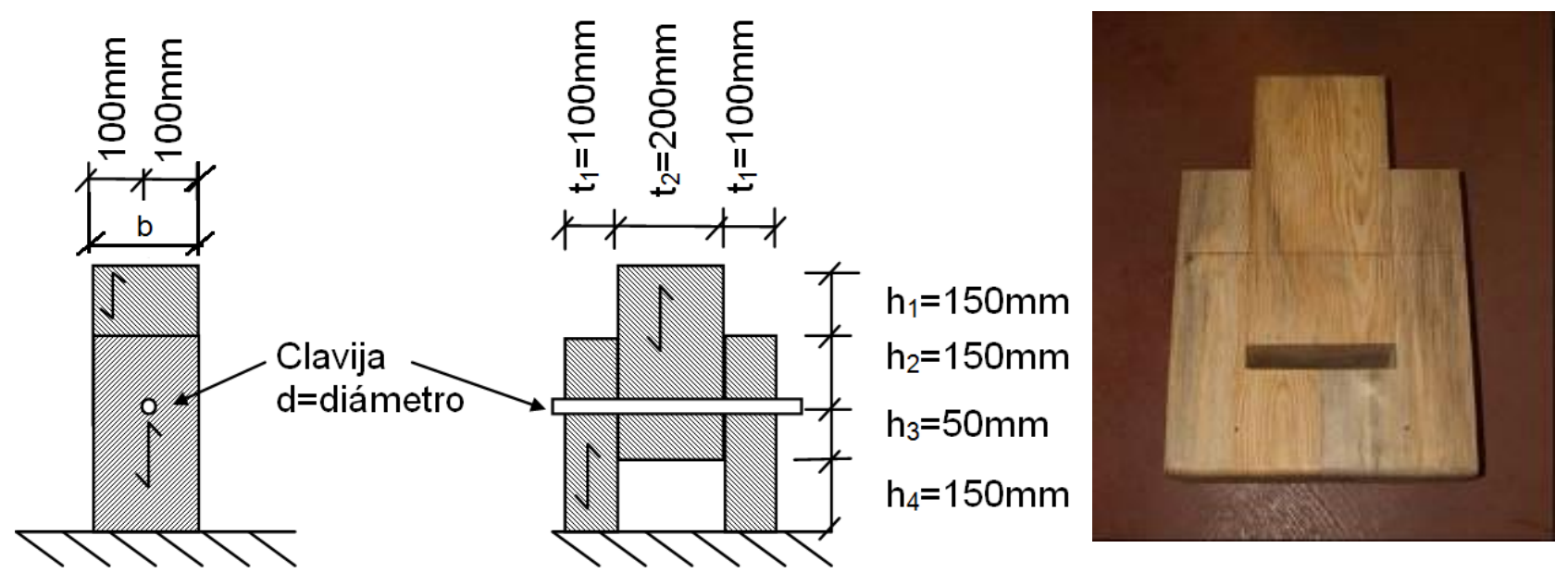

Fig. 5: Dimensiones y geometría de las uniones ensayadas

En estos ensayos ha sido muy importante analizar la aparición de rótulas plásticas en la clavija según los modos de fallo iii) y iv) de la figura 1 y el aplastamiento en las piezas de madera según los modos de fallo i) y ii) de la misma figura. En los primeros ensayos, las fibras estaban alineadas con la carga que provoca el fallo según muestra la figura 6 . También son interesantes estudios con diferentes ángulos $(\alpha)$, entre la línea de carga y las fibras de la madera, fijando una rotación en la pieza central de madera hasta llegar a orientaciones de $90^{\circ}$. Con ello se pueden estudiar los efectos de la ortotropía, según la figura 6. 

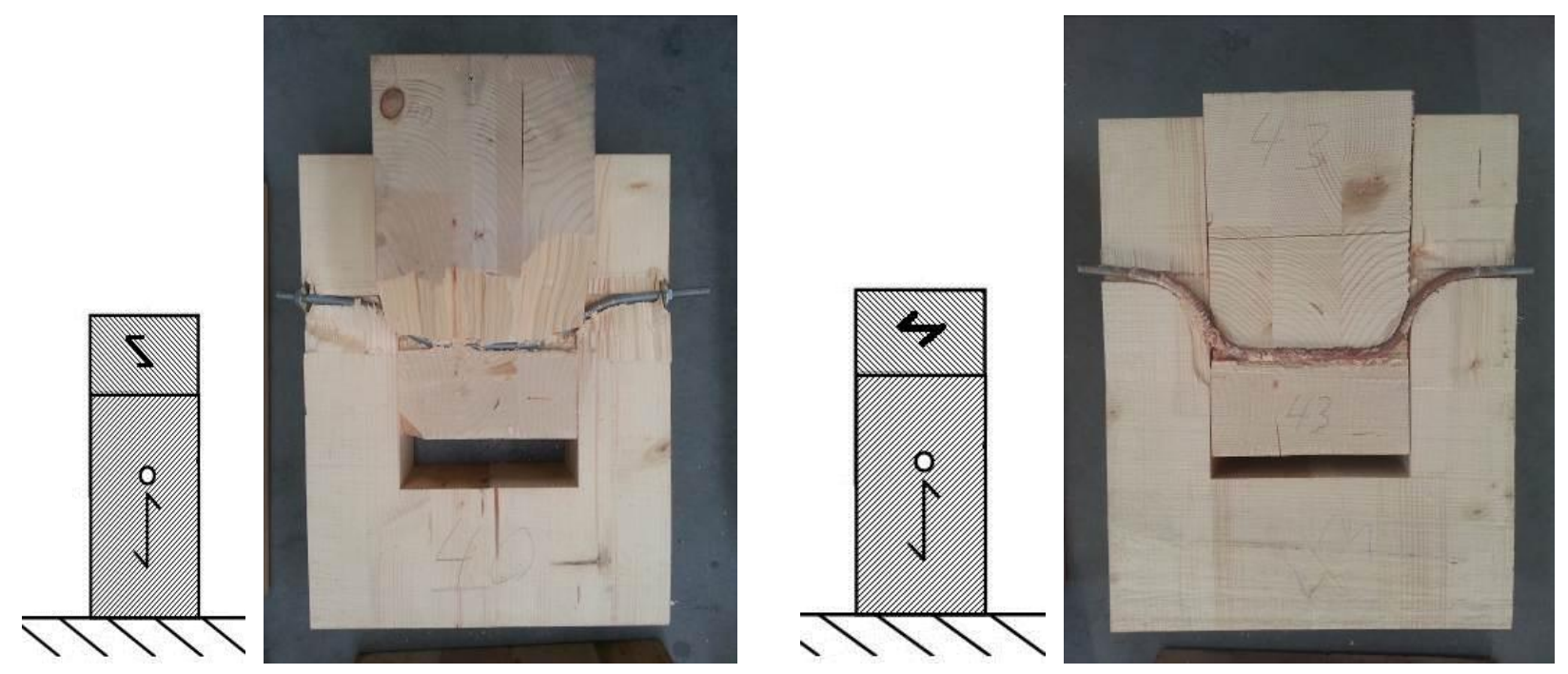

Fig. 6: Probetas con pieza central orientada a 45 y $90^{\circ}$

\section{Número de probetas}

Tomando como referencia las exigencias de propiedades mecánicas de madera para uso estructural, cada muestra debe consistir en un grupo de probetas con características iguales, de los tamaños más representativos (escuadría y longitud) que se pueden obtener para una especie y procedencia. Estos ensayos deben realizarse sobre muestras como mínimo de 40 probetas según normas como la UNE EN 384 y son penalizadas con un factor ks (en función del número y tamaño de las muestras) aplicado sobre el valor final de la resistencia característica en la madera, donde $A$ es el número de probetas de cada muestra (de igual sección transversal) y $B$ es el número total de probetas. Para no sufrir estas penalizaciones se necesitarían del orden de 200 probetas según indica la figura 7.

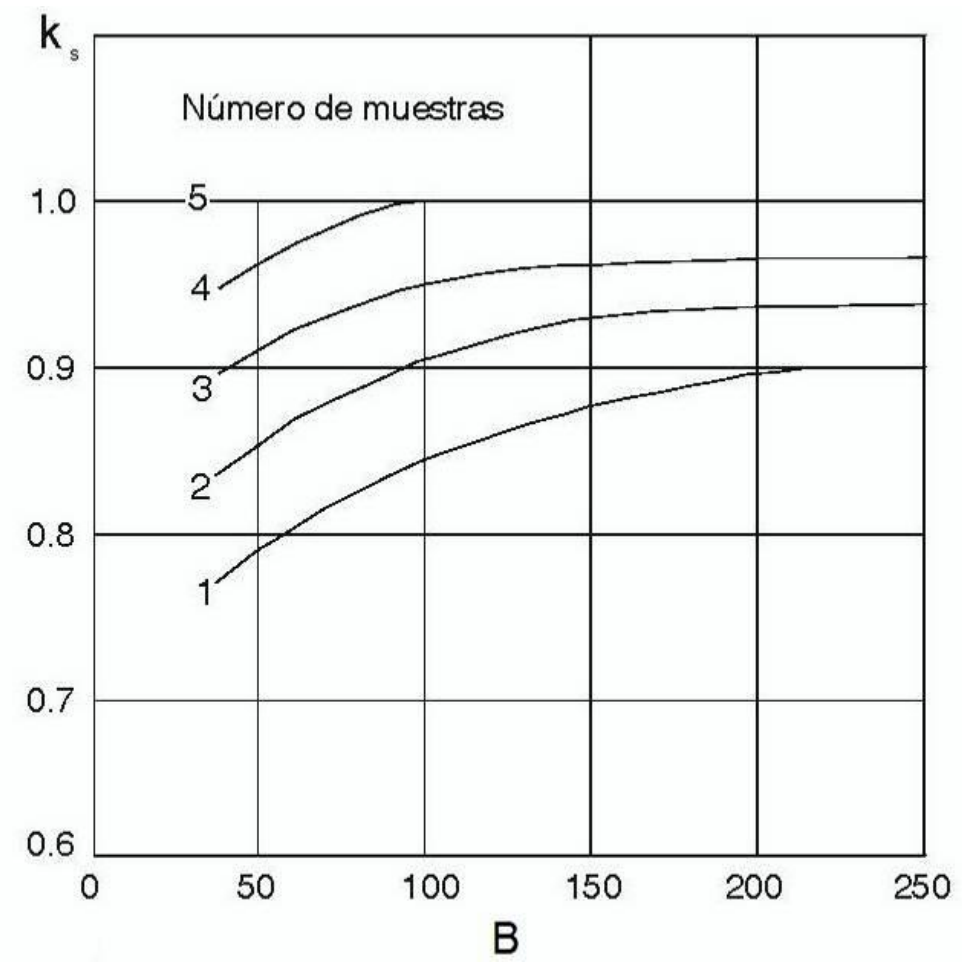

Fig. 7: Determinación del coeficiente $\mathrm{k}_{\mathrm{s}}$

En los procedimientos de ensayo desarrollados hasta la fecha, se fijan muestras con tres probetas iguales, llegando a realizar 132 probetas con variaciones de ancho ( $\left.\mathrm{t}_{1}\right)$, diámetro (d), encolado y arandela y orientaciones de fibra desde $0^{\circ}$ a $90^{\circ}$. 


\section{Comportamiento mecánico del conjunto.}

El grado de rigidez de la unión es un factor a considerar sobre todo en uniones articuladas. Este depende de un gran número de factores, algunos de los cuales están fuera del control del proceso de diseño y cálculo. Las hipótesis que dan lugar al dimensionado teórico, por ejemplo, la de articulación perfecta en el nudo, pueden estar expuestas a variables tales como excentricidades de agujeros, separación de intercaras de madera, distribución de cargas iniciales sobre el nudo que deriven en deslizamientos, etc. Es de especial interés la hipótesis para el desarrollo de las ecuaciones analíticas de fallo de la clavija (Johansen, 1949), que parten del comportamiento rígido-plástico en la clavija mostrado en la figura 8. La determinación de métodos que den garantías del comportamiento a rigidez real de una forma fiable puede suponer una base para determinar técnicas de mejora del nudo así como la relación con las propuestas analíticas, lo cual hace orientar el trabajo a determinar curvas de carga frente a deformación y calcular el área, a modo de trabajo que puede absorber la unión.

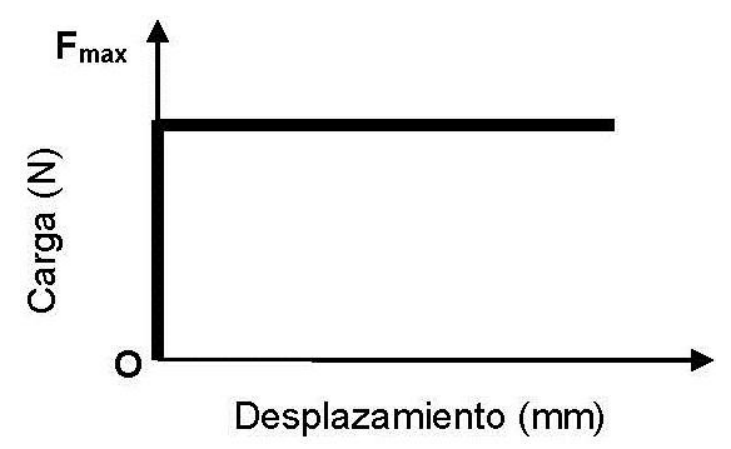

Fig. 8: Simplificación de la curva carga-deformación

\section{RESULTADOS}

Sobre la preparación de las probetas y ensayos se define un informe particular para cada probeta y otro de la muestra con los valores del $5^{\circ}$ percentil, en él se muestran las características significativas de la madera, las propiedades de los materiales y elementos de fijación, sus dimensiones, el acondicionado de la madera y de las probetas antes y después de la fabricación, (humedad, fendas y defectos de inspección visual) el procedimiento de carga empleado, cualquier información pertinente relacionada con los ajustes valores medios y desviaciones normalizadas, así como la descripción del modo de fallo según lo indicado en la figura 1.

Las curvas representadas en la figura 9 permiten identificar el comportamiento de los distintos tipos de uniones con clavija. Dichas gráficas corresponden a las relaciones carga-desplazamiento obtenidas en los ensayos con las probetas de dimensiones tipo $t_{1}=100 \mathrm{~mm}, t_{2}=200 \mathrm{~mm}, \alpha=0^{\circ}$ y $d=10 \mathrm{~mm}$, para las distintas variantes de clavija consideradas (bulón roscado, con adhesivo y con fijación arandela-tuerca), así como los resultados del cálculo según las ecuaciones analíticas. La UNE EN 26891 marca la carga máxima para un deslizamiento fijo de $15 \mathrm{~mm}$ y puntos característicos del ensayo. En el caso de la figura 9, la muestra con adhesivo admite cargas superiores, pero una vez perdida la adherencia del mismo, los valores resistentes decrecen para aproximarse al ensayo con clavija simple, mientras que el conjunto con tuerca arandela sigue incrementando la carga. Estas variaciones en la pendiente carga - deslizamiento llevan a integrar la función. Con ello se determina la energía adsorbida en la unión. La composición de la gráfica real en el informe de ensayo también resulta importante, porque definir solo puntos característicos (especialmente si se dan pendientes negativas), puede enmascarar el comportamiento provocado por los elementos de refuerzo.

Se observa que el empleo de adhesivo y de tuerca-arandela, a pesar de que existe cierta región de plastificación de la madera, permite alcanzar valores superiores de la carga. Por lo tanto, el comportamiento con adhesivo y tuerca-arandela es superior, incluido el analítico recogido en Eurocódigo 5. La gráfica de la unión con adhesivo muestra tramos cuasi elásticos con valores que superan del orden de cuatro veces la resistencia de cálculo; con lo cual, las ecuaciones analíticas de Johansen, que responden a una línea horizontal que muestra la figura 9 , están muy del lado de la seguridad en uniones de este tipo. 


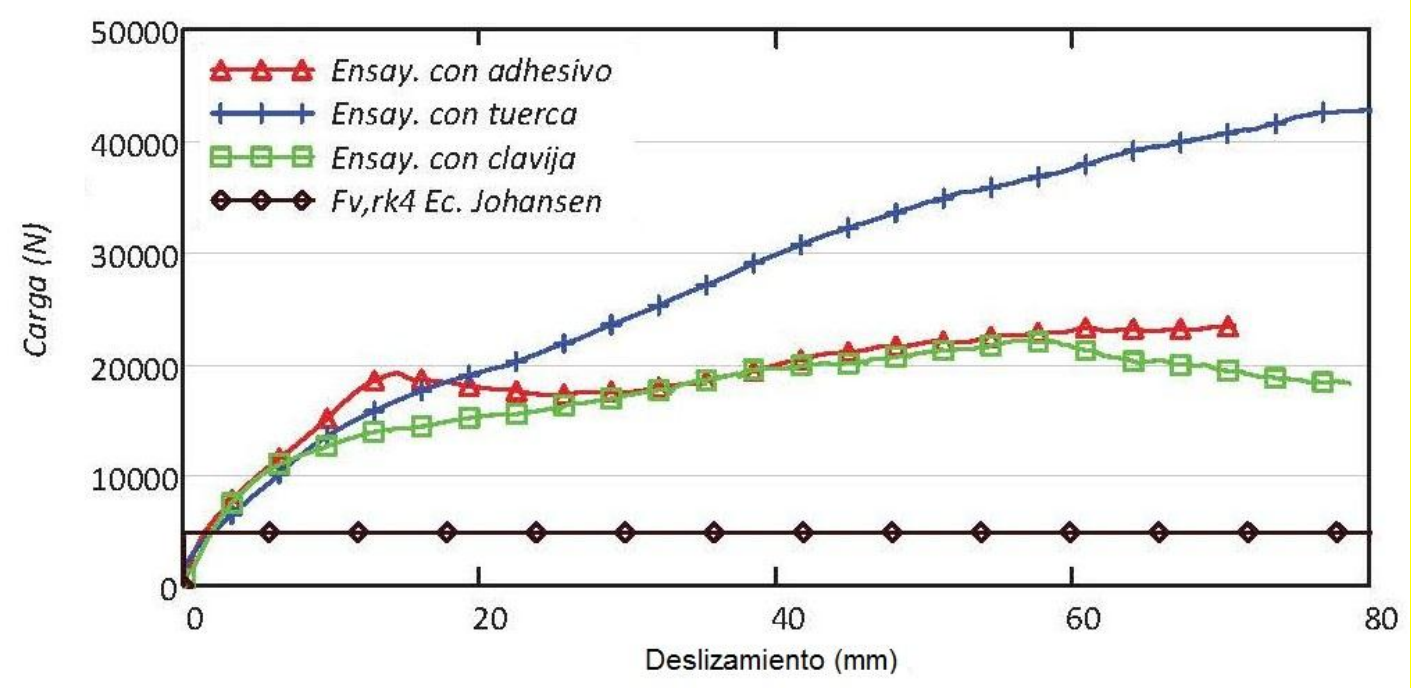

Fig. 9: Comparativa entre distintas tipologías de unión (régimen plástico)

Tras ejecutar el ensayo es aconsejable realizar un análisis de la sección transversal de la probeta tal y como se observa en la figura 10. Esto permite identificar las rótulas plásticas producidas (que condicionan la ecuación analítica de comparación) y las superficies de aplastamiento que se han producido en la clavija (si es que han tenido lugar) lo cual permite determinar el modo de fallo real ocurrido de entre los identificados en la figura 1. Con estas secciones se puede analizar el grado de aplastamiento en la madera, lo que requiere fijar el desplazamiento en los ensayos en un límite superior, aunque esto limita la información de la curva carga desplazamiento.

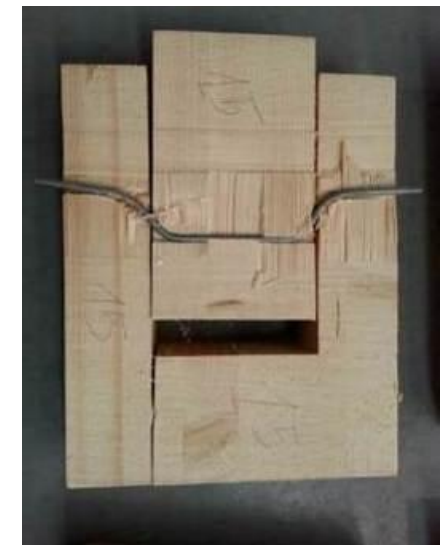

Clavija

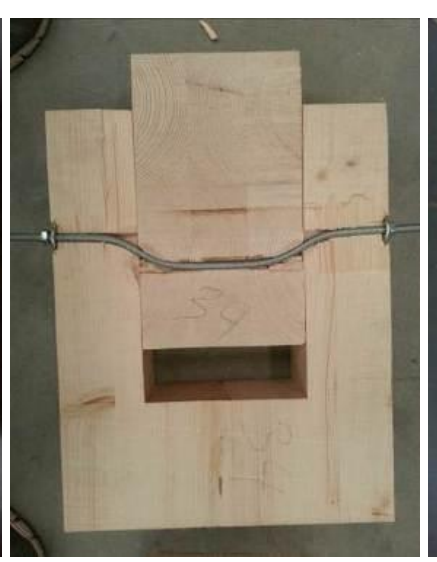

b) roscada con tuerca arandela

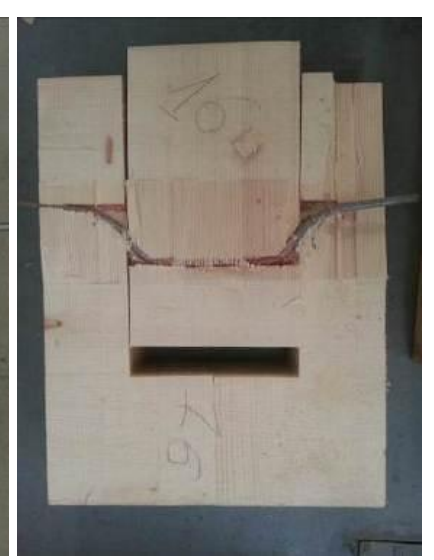

c) roscada encolada

Fig. 10: Probetas cortadas transversalmente tras el ensayo

La unión con tuerca - arandela mantiene su resistencia incluso para grandes deformaciones y absorbe gran cantidad de energía al deformar la madera mediante el efecto soga, definido en DB SE-M para clavos y tirafondos, y muy probablemente por la fricción superficial con la madera que induce en las superficies de deslizamiento un comportamiento cuasi elástico lineal en las primeras fases de carga.

\section{CONCLUSIONES}

De los resultados mostrados, de su análisis y de su discusión, se pueden obtener las siguientes conclusiones:

La existencia de una gran cantidad de variables en el diseño de uniones clavija supone fijar parte de las características de las probetas y un número mínimo de ensayos penalizando los resultados cuando el número de probetas sea pequeño. Las condiciones termo higrométricas de ensayo deben ser medidas y en especial la humedad de la madera, si sobrepasa el $12 \%$, su resistencia también debe ser penalizada. 
En el trabajo se fijan condiciones para los ensayos en velocidad de desplazamiento, límites de deslizamiento y posiciones geométricas para evitar fallos no previstos, como la aparición de hiendas. Es muy aconsejable que, tanto la madera como la clavija, respondan a dimensiones y materiales bien caracterizados de forma independiente, preferentemente con certificados de calidad. Se deben hacer controles previos de cada uno de los elementos orientados a ratificar las propiedades de las partes.

El fijar un deslizamiento máximo, 15 mm según la norma UNE EN 26891, o puntos característicos del ensayo, debe ser complementado con las particularidades de cada elemento añadido a la unión. La caída de carga al perder la adherencia el epoxi supone una mayor fiabilidad de la clavija con tuerca y arandela para deslizamientos mayores.

Los resultados de los ensayos realizados muestran unos valores superiores a los que se obtendrían mediante las formulas analíticas contenidas en la normativa, lo que demuestra que el modelo analítico rígido-plástico, adoptado en las normas, difiere del comportamiento real del nudo por el lado de la seguridad.

En futuros trabajos, parece interesante la aplicación de superposición de efectos, para identificar funciones de ajuste a las distintas gráficas.

\section{AGRADECIMIENTOS}

Este trabajo ha sido realizado gracias al proyecto de investigación subvencionado por la Junta de Castilla y León de código SA066A08.

\section{REFERENCIAS}

Argüelles, R., F. Arriaga y J. J. Martínez, Estructuras de madera. Diseño y cálculo, 2ª edición, 307-320, AITIM, Madrid, España (2003).

Ariza, I., El nuevo enfoque en los ensayos mecánicos de la madera aserrada para uso estructural en la normativa europea, Madera y Bosques 8(2), 3-16 (2002).

Awaludin, A., T. Hirai, T. Hayashikawa e Y. Sasaki, Load-carrying capacity of steel-to-timber joints with a pretensioned bolt, J Wood Sci, 54, 362- 368 (2008).

Bainbridge, R., C. Mettem, K. Harvey y M. Ansell, Bonded-in rod connections for timber structures development of design methods and test observations, International Journal of Adhesion \& Adhesives, 22, 47-59 (2002).

Batchelar, ML. y KA. Mcintosh, Structural joints in glulam, Proceedings of the 5th World conference in Timber Engineering, Montreux, 4(7), 289-296 (1988).

Blanchet, P., Caractérisation du comportement des lames de plancher d'ingénierie, Tesis doctoral, Faculté de foresterie et de géomatique. Université Laval, Québec-Canadá, (2004).

Cárdenas M., F. Schanack y O. R. Ramos, Diseño, construcción y ensayo de una estructura de sección mixta madera laminada-hormigón para su uso en puentes, Revista de la Construcción, 9(2), 63-75 (2010).

CTE, DB SE-M, Código técnico de la edificación. Documento básico. Seguridad estructural madera, Madrid, España (2009).

Davis T. J. y P. A. Claisse, Bolted joints in glulam and structural timber composites, Construction and Building Materials, 14, 407-417 (2000).

Dong, L, Développement de modèles analytiques pour la prédiction du comportement élastique des assemblages mécaniques à broches dans la construction en bois, Tesis doctoral, Faculté des Sciences Appliquées Génie Civil et Géologie, Université de Liège, Bélgica (2006).

Dorn, M., K. Borst y J. Eberhardsteiner, Experiments on dowel-type timber connections, Engineering Structures, 47, 67-80 (2013). 
Echavarría C.A., C.A. Correa y C. Echevarri, Conexiones pernadas de madera reforzadas con placas dentadas de acero, Dyna 164, №. 164 98-108 (2009).

Fueyo, J.G., M. Domínguez y J.A. Cabezas, Rendimiento de uniones clavija de tipo perno encolado usando elementos finitos, Información Tecnológica (CIT) 117-128 (2011).

Guan Z.W. y P.D. Rodd, Hollow steel dowels - a new application in semi-rigid timber connections, Engineering Structures 23, 110-119 (2001).

Hermoso E., J. Carballo y J.I. Fernández-Golfín, Caracterización estructural de la madera de pinus radiata d. Don del Pais Vasco (España) acorde a las modificaciones normativas, Maderas. Ciencia y tecnología, 9(3), 223-232 (2007).

Hilson, B.O., Joints with dowel-type fasteners, Theory. Timber Engineering, STEP 1. Centrum Hout. Holanda (1995).

Íñiguez, G., Clasificación mediante técnicas no destructivas y evaluación de las propiedades mecánicas de la madera aserrada de coníferas de gran escuadría para uso estructural, Tesis doctoral, Departamento de construcción y vías rurales, Universidad Politécnica de Madrid, Madrid-España (2007).

Johansen, K.W., Theory of timber connections, International Association of Bridge and Structural Enginnering, 9, 249-262 (1949).

Larsen, H.J. y J.L. Jensen, Influence of Semi-rigidity of Joints on the Behaviour of Timber Structures, Progress In Structural Engineering And Materials, 2, 267-277 (2000).

Rodd P.D. y Leijten A.J.M., High-performance dowel-type joints for timber structures Progress In Structural Engineering And Materials, 5, 77-89 (2003).

UNE EN 338, Madera estructural. Clases resistentes, AENOR, Comité técnico AEN/CTN 56 Madera y Corcho, 1-16, Madrid, España (2003).

UNE EN 383, Estructuras de madera. Métodos de ensayo. Determinación de la resistencia al aplastamiento y del módulo de aplastamiento para los elementos de fijación tipo clavija, AENOR, Comité técnico AEN/CTN 56 Madera y Corcho, 1-16, Madrid, España (2007).

UNE EN 384, Madera con uso estructural. Determinación de los valores característicos de las propiedades mecánicas y densidad, AENOR, Comité técnico AEN/CTN 56 Madera y Corcho, 1-18, Madrid, España (2004).

UNE EN 408, Estructuras de madera. Madera aserrada y madera laminada encolada para uso estructural. Determinación de algunas propiedades físicas y mecánicas, AENOR, Comité técnico AEN/CTN 56 Madera y Corcho, 1-36, Madrid, España (2004).

UNE EN 1995, Eurocódigo 5, Proyecto de estructuras de madera, AENOR, Comité técnico AEN/CTN 56 Madera y Corcho, 1-138, Madrid, España (2006).

UNE EN 26891, Estructuras de madera. Uniones realizadas con elementos de fijación mecánicos. Determinación de las características de resistencia y deslizamiento, AENOR, Comité técnico AEN/CTN 56 Madera y Corcho, 1- 10, Madrid, España (1992).

UNE 56544, Clasificación visual de la madera aserrada para uso estructural. Madera de coníferas, AENOR, Comité técnico AEN/CTN 56 Madera y Corcho, 1-26, Madrid, España (2011).

USDA, Wood Handbook: Wood as an Engineering Material, $1^{\text {a }}$ edición, 7.1-7.28, Fastenings USDA Forest Service, Forest Products Laboratory, Madison, WI (1998). 